\title{
Factors Affecting Pulse Injection from Measuring Electrode Based on the Pulsed Electroacoustic Technology for Coaxial Cables
}

\author{
Wang Xia, ${ }^{*}$ Hao Jia-Qi, Xiong Jin-Zhou, Wu Kai, Fu Ming-Li, ${ }^{1}$ and Hou Shuai ${ }^{1}$ \\ State Key Laboratory of Electric Insulation and Power Equipment, Xi'an Jiaotong University, \\ Xi'an 710049, China \\ ${ }^{1}$ Electric Power Research Institute, China Southern Power Grid, Guangzhou 510080, China
}

(Received December 1, 2016; accepted March 23, 2017)

Keywords: pulsed electroacoustic method, coaxial cable, coupled pulse, pulse injection, outer semiconductive layer

In the pulsed electroacoustic (PEA) space charge measurement system used for coaxial cables, the waveform of a coupled pulse will directly affect space charge profiles in cable insulation. In this paper, on the basis of the PEA measurement system with pulse injection from a measuring electrode, the original charge acoustic signals in a $35 \mathrm{kV}$ cross-linked polyethylene (XLPE) cable were collected directly from the oscilloscope under different ground distances between the measuring point and the ground point along a cable's semiconductive (SC) layer. At the same time, the effects of ground distance, cable specification, and cable length on the waveform of a coupled pulse were studied through a series of experiments and simulations. The results show that the amplitude of the coupled pulse is directly proportional to the ground distance but inversely proportional to the cable cross section or voltage class. Furthermore, the pulse coupling efficiency is almost the same only if the cables have the same diameter of the outer SC layer. Therefore, a constant pulse coupling efficiency can be obtained by adjusting the ground distance according to the diameter of the cable's outer SC layer.

\section{Introduction}

With the advantages of high transmission capacity, low loss, difficult to age, long lifetime and unlimited transmission distance, high-voltage direct-current (HVDC) cable transmission has many prospects in electrical fields, such as long-distance and high-capacity transmission, power system interconnection, DC cable transmission and alternating current (AC) cable capacity expansion. ${ }^{(1-3)}$ However, the effect of space charge is the bottleneck during the development of HVDC plastic cables. $^{(4-6)}$ Until now, many studies at home and abroad have shown that the existence, transfer and disappearance of space charge will directly lead to the distortion of the local electric field in materials and then further indirectly affect the conductivity, breakdown, aging and other aspects of insulating materials. ${ }^{(7-11)}$

The pulsed electroacoustic (PEA) technology is one of the commonly used nondestructive methods of space charge measurement in a solid dielectric. To date, some scholars have carried

"Corresponding author: e-mail: wxflying@mail.xjtu.edu.cn http://dx.doi.org/10.18494/SAM.2017.1510 
out studies on the space charge profiles in the coaxial cable based on the PEA method and have achieved many preliminary results. ${ }^{(12-18)}$ According to the high-voltage (HV) pulse injection mode, the PEA space charge measurement method used for the coaxial cable can be divided into three types: (i) pulse injection from an HV conductor, ${ }^{(12-15)}$ (ii) pulse injection from a partially stripped outer semiconductive (SC) layer, ${ }^{(16)}$ and (iii) pulse injection from a measuring electrode. ${ }^{(17,18)}$ Among them, the 3rd type with pulse injection from a measuring electrode can fulfill the space charge measurement for large and long cables, which can not only overcome the corona discharge and high-frequency pulse attenuation in the 1st type but also avoid the damage of the cable's outer SC layer in the 2nd type.

In the 3rd type, to enhance the space charge signal in the coaxial cable, the HV pulse injects from the measuring electrode of the PEA system firstly and then couples into a cable conductor via the cable's bulk capacitor. Thus, the HV pulse injection is the key step during space charge measurement. However, when the HV pulse is injected from the PEA measuring electrode, the coupled pulse propagates not only through the bulk capacitor of the cable but also along the outer $\mathrm{SC}$ layer. However, until now, few researchers have paid attention to the factors affecting the resistance of the outer SC layer and its effect on pulse coupling efficiency.

In this study, on the basis of the PEA measurement system with pulse injection from a measuring electrode, the original charge acoustic signals in a $35 \mathrm{kV}$ cross-linked polyethylene (XLPE) cable were collected directly from the oscilloscope under different ground distances between the measuring point and the ground point along the outer SC layer. Then, the effects of ground distance, cable specification and cable length on the waveform of a coupled pulse were studied through a series of experiments and simulations. Finally, a criterion for the selection of ground distance was proposed to fulfill the constant amplitude of the coupled pulse when this PEA method was used for actual long cables.

\section{Experimental Methods}

\subsection{Test cables}

The test cables used in this study were 10,35, and $110 \mathrm{kV}$ AC XLPE cables. The length of each cable was about $1.33 \mathrm{~m}$. For the coaxial cable, the capacitance of the cable insulation or the resistance of the outer SC layer per unit length is given by the capacitance of cable insulation,

$$
C_{0}=2 \pi \varepsilon_{0} \frac{\varepsilon_{r}}{\ln \left(r_{2} / r_{1}\right)}
$$

and the resistance of the outer SC layer,

$$
R_{0}=\rho /\left[\pi\left(r_{3}^{2}-r_{2}^{2}\right)\right]=\rho /\left[\pi\left(r_{3}-r_{2}\right)\left(r_{3}+r_{2}\right)\right]
$$

Here, $\varepsilon_{0}$ is the vacuum dielectric constant, $\varepsilon_{r}$ is the relative permittivity of the dielectric, $\rho$ is the dielectric resistivity, $r_{1}$ and $r_{2}$ are the inner and outer radii of cable insulation, respectively, and $r_{3}$ is the outer radius of the outer SC layer of the cable. In addition, the conductivity of the outer SC layer is about $2.0 \mathrm{~S} / \mathrm{m}$ at the high frequency of megahertz. ${ }^{(19)}$

According to Eqs. (1) and (2), the electrical parameters of the three types of XLPE cable are obtained, as shown in Table 1. 
Table 1

Electrical parameters of experimental cables at $10 \mathrm{MHz}$.

\begin{tabular}{lccc}
\hline \multirow{2}{*}{\multicolumn{1}{c}{ Item }} & \multicolumn{3}{c}{ Cable } \\
\cline { 2 - 4 } & $10 \mathrm{kV}\left(70 \mathrm{~mm}^{2}\right)$ & $35 \mathrm{kV}\left(300 \mathrm{~mm}^{2}\right)$ & $110 \mathrm{kV}\left(800 \mathrm{~mm}^{2}\right)$ \\
\hline Diameter of inner SC layer $(\mathrm{mm})$ & 9.4 & 21.0 & 39.8 \\
Diameter of insulation $(\mathrm{mm})$ & 18.4 & 41.0 & 70.8 \\
Diameter of outer SC layer $(\mathrm{mm})$ & 20.0 & 42.8 & 73.8 \\
Capacitance of cable insulation $(\mathrm{pF} / \mathrm{m})$ & 190.4 & 191.2 & 222.0 \\
Resistance of outer SC layer $(\mathrm{k} \Omega / \mathrm{m})$ & 10.4 & 4.2 & 1.5 \\
\hline
\end{tabular}

\subsection{Space charge measurement}

Figure 1 shows the PEA measurement system with pulse injection from a measuring electrode. The device consists of an HVDC power supply, a nanosecond pulse, a set of measuring electrodes, an oscilloscope and a computer. The HVDC source can provide a $60 \mathrm{kV}$ DC high voltage and the pulse source can produce a periodic nanosecond pulse with a width of $200 \mathrm{~ns}$ and a maximal voltage of $20 \mathrm{kV}$. The measuring electrode includes a measuring unit and a cable clamping unit. Here, the maximum acquisition frequency of the oscilloscope is $5 \mathrm{GHz}$.

The measuring unit consists of an aluminum electrode, a polyvinylidene fluoride (PVDF) piezoelectric sensor, a sound absorber and an amplifier. The HVDC power supply is used to apply voltage to the cable during measurement. The pulse injects from the measuring electrode and then couples into a cable conductor via the cable's bulk capacitor. Thus, besides the equipotential oscilloscope, the measuring electrode is insulated from the ground by an epoxy column and powered by a battery. The isolation between the oscilloscope and the computer is fulfilled through a digital optical fiber. ${ }^{(18)}$ The computer is used to remotely control the oscilloscope and to realize waveform acquisition and data processing.

The measurement methods of space charge in the cables were as follows:

(i) Comparison and analysis of different pulse injection methods. In view of the limitation of the applied voltage, the original charge acoustic signals in the $10 \mathrm{kV}$ XLPE cable were measured by the methods of pulse injection from the HV conductor and measuring electrode.

(ii) Different ground distances between the measuring and ground points along the outer SC layer. The original charge acoustic signals in the $35 \mathrm{kV}$ XLPE cable were measured by the method of pulse injection from the measuring electrode under different ground distances.

During the above experiments, the amplitude of the nanosecond pulse source was $2 \mathrm{kV}$. The DC voltage of $15 \mathrm{kV}$ was applied for $20 \mathrm{~min}$.

\subsection{Coupled pulse measurement}

In this study, a high-voltage pulse source with a maximal amplitude of $20 \mathrm{kV}$ and a pulse width of $200 \mathrm{~ns}$ was built using the high-frequency solid-state switch (HTS 50-08-UF). ${ }^{(16)}$ Figure 2 shows the circuit of the HV pulse source, and point A represents the measurement point of the pulse output waveform.

Figure 3 shows the measured HV pulse waveform. It can be seen from Fig. 3 that the amplitude of the output pulse is about $2 \mathrm{kV}$ and the pulse width is about $200 \mathrm{~ns}$.

Connected to the measuring electrode of the PEA system, the pulse injects into a cable from 


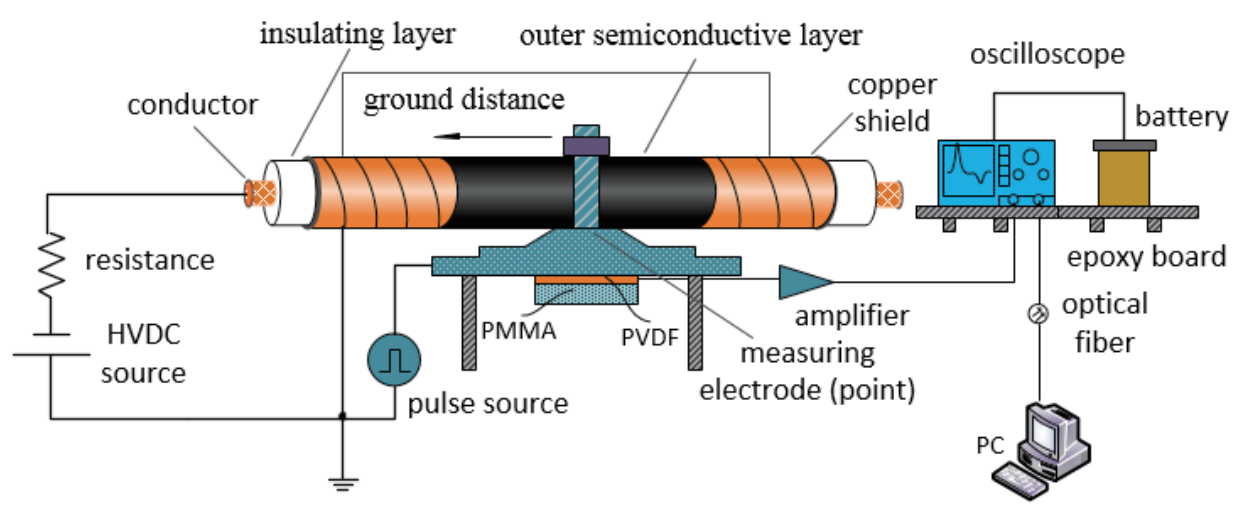

Fig. 1. (Color online) Schematic diagram of PEA system for cable geometry.

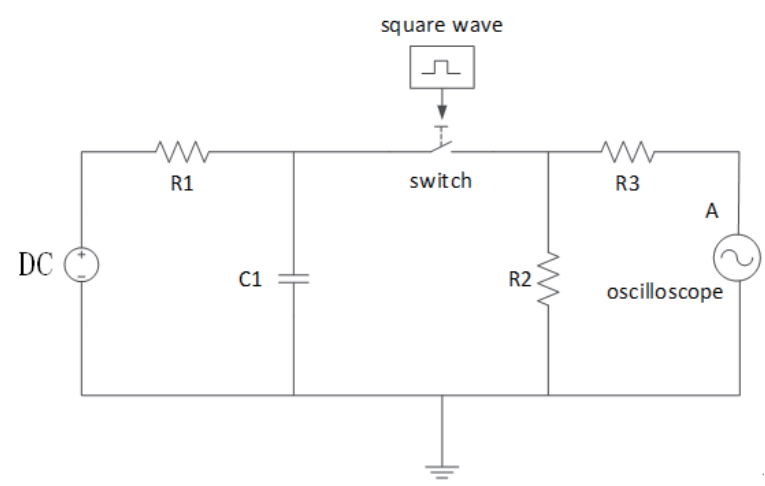

Fig. 2. Circuit diagram of HV pulse generator.

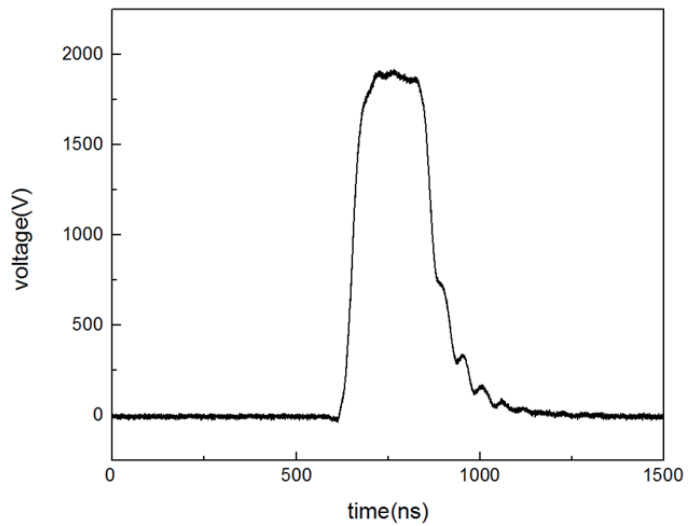

Fig. 3. Measured HV pulse waveform.

the outer SC layer. The schematic diagram of the pulse injection from the outer SC layer is shown in Fig. 4(a). Figure 4(b) shows its equivalent circuit, which will be used for simulation. In Fig. 4(a), the middle outer SC layer is coated with an aluminum foil segment acting as the measuring or pulse injection point [i.e., point B in Fig. 4(b)]. The length of the exposed outer SC segments after stripping the copper shield is defined as the ground distance. In Fig. 4(b), $C_{x}$ is the capacitance of cable insulation at the measuring point, $C_{0}$ is the distributed capacitance of cable insulation, $R_{g}$ is the distributed resistance of the outer SC layer, and point B represents the measuring point of the coupled pulse.

As shown in Fig. 4, when the pulse injects from the measuring point, the coupled pulse propagates not only through the cable's bulk capacitor but also along the outer SC layer. This means that the resistance of the outer SC layer as well as the cable capacitance will affect the pulse coupling efficiency. Therefore, the measurement methods of the coupled pulse were as follows:

(i) Amplitude of coupled pulse with the variation of ground distance. Here, the ground distance changed from 2 to $17 \mathrm{~cm}$ during the measurement.

(ii) Amplitude of coupled pulse with the variation of cable specification. The three XLPE cables with different voltage classes in Table 1 were selected as test objects. Here, the ground distances were all fixed at $17 \mathrm{~cm}$. 


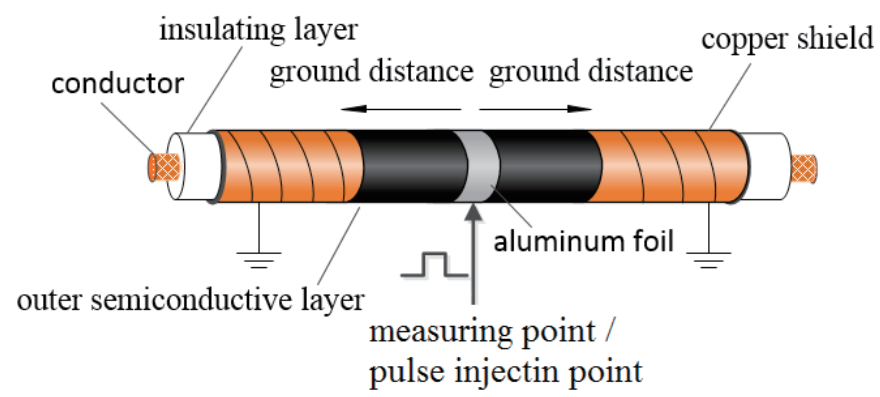

(a)

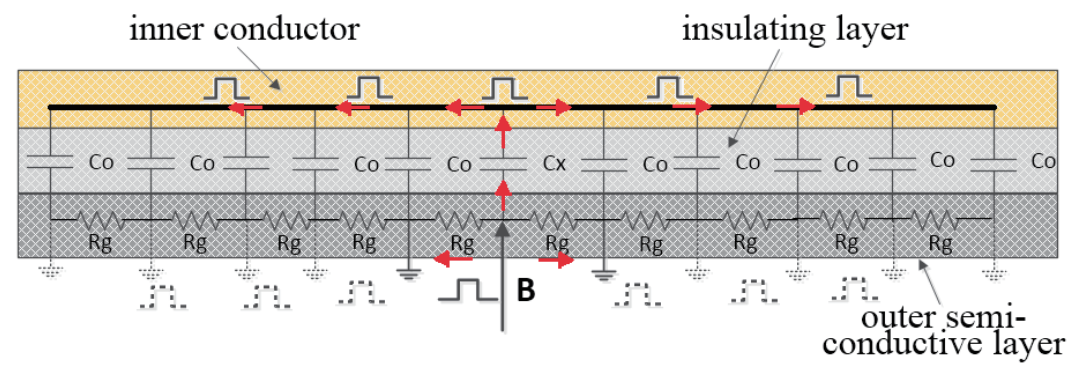

(b)

Fig. 4. (Color online) Diagram of pulse injection from the outer SC layer. (a) Schematic diagram. (b) Equivalent circuit.

\subsection{Simulation and analysis of the coupled pulse}

Pspice software was used to model and simulate the pulse source shown in Fig. 2. Figure 5 shows the simulation result of the nanosecond pulse source waveform, which is almost in agreement with the measured waveform shown in Fig. 3.

According to the equivalent circuit shown in Fig. 4(b), the simulation methods of the coupled pulse waveform were as follows:

(i) Verification of test results. On the basis of the cables in Table 1, the amplitudes of the coupled pulse were simulated by changing the ground distance and cable specification.

(ii) Relationship among pulse coupling efficiency, cable length and ground distance. In view of the actual long cable, the amplitudes of the coupled pulse were simulated by changing the cable length and cable specification.

\section{Experimental Results and Simulation Analysis}

\subsection{Space charge measurement}

In order to declare the validity of measurement results, the traditional PEA measurement method with pulse injection from the cable's HV conductor was introduced as a reference. Figure 6 shows the original charge acoustic signals collected directly from the oscilloscope in the $10 \mathrm{kV}$ XLPE. Here, the broken lines represent electrode sites, the left one is the outer insulation layer, and the right one is the inner insulation layer. 


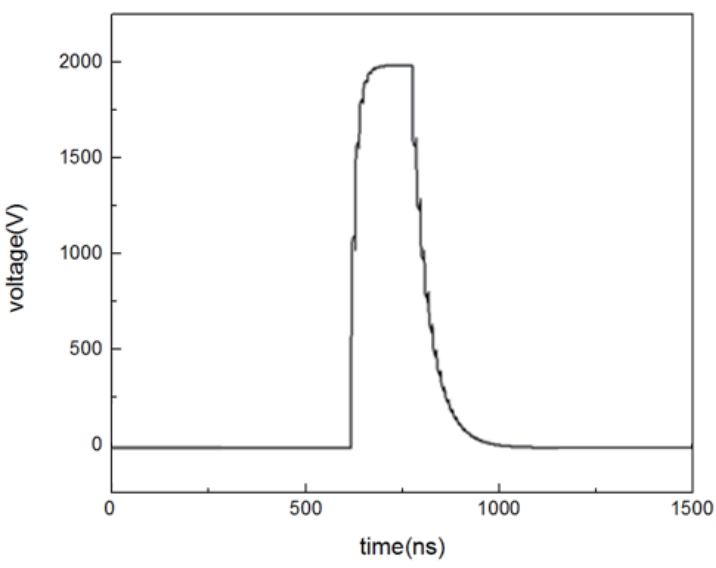

Fig. 5. Simulated HV nanosecond pulse waveform.

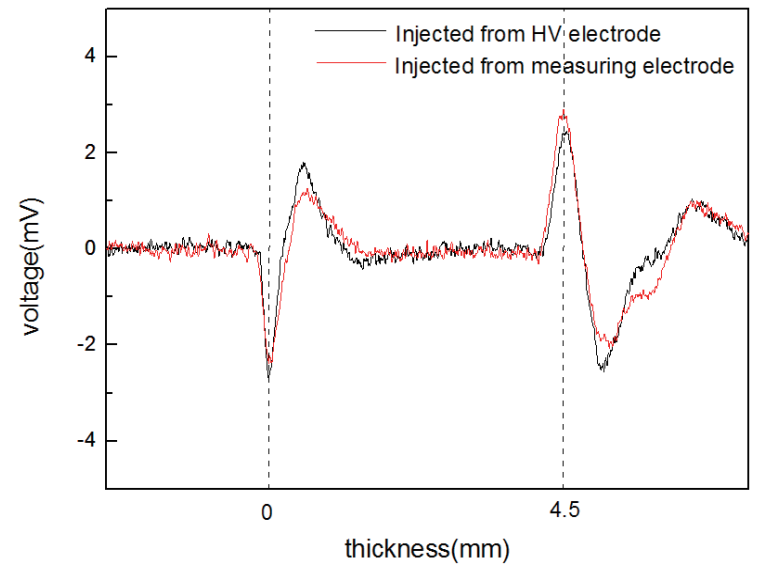

Fig. 6. (Color online) Original charge acoustic signals in the $10 \mathrm{kV}$ XLPE cable with different pulse injection methods.

As shown in Fig. 6, the waveform measured by the method of pulse injection from the measuring electrode is basically consistent with that from the HV electrode. Hence, this PEA method with pulse injection from the measuring electrode can also effectively measure the space charge characteristics in the cable.

Figure 7 shows the original charge acoustic signals in the $35 \mathrm{kV}$ XLPE cable under different ground distances. It can be seen from Fig. 7 that the amplitude of the acoustic signal increases gradually when the distance between the ground point and the pulse injection point increases from 2 to $17 \mathrm{~cm}$.

However, it is worth mentioning that, in this paper, we mainly emphasize the effect of the pulse injection mode on the charge signal waveform. Thus, it is the reason why the waveforms shown in Figs. 6 and 7 are all the original charge acoustic signals collected from oscilloscope without the waveform recovery and calibration.

\subsection{Relationship between amplitude of coupled pulse and ground distance}

To verify the effects of ground distance on the amplitude of the coupled pulse, the coupled pulse waveform was measured synchronously during the space charge measurement. Figure 8(a) shows the coupled pulse waveform in the $35 \mathrm{kV}$ XLPE cable under different ground distances. It can be seen from Fig. 8(a) that the amplitude of the coupled pulse decreases from $1.5 \mathrm{kV}$ to $500 \mathrm{~V}$ when the distance between the ground point and the pulse injection point decreases from 17 to $2 \mathrm{~cm}$. Here, the voltage of the pulse source in the experiment and simulation is $2 \mathrm{kV}$. Figure 8 (b) shows the simulation results of the coupled pulse according to Fig. 4 and the cable parameters in Table 1. It can be found that the test results in Fig. 8(a) are basically similar to the simulation results in Fig. 8(b).

From the foregoing, the amplitude of the coupled pulse is closely related to the ground distance along the outer SC layer. This means that the variation of the ground distance will result in the change in the amplitude of the coupled pulse at the pulse injection point (Fig. 8) and then in the change in charge acoustic signal intensity (Fig. 7). 


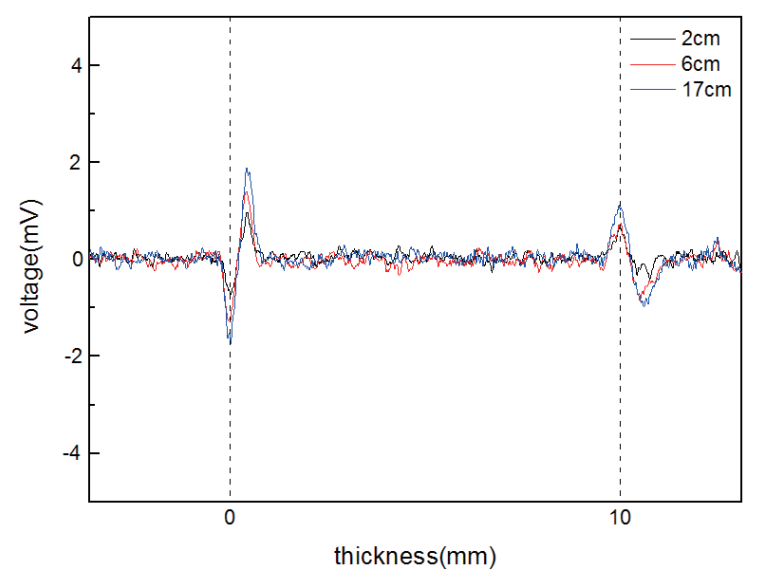

Fig. 7. (Color online) Original charge acoustic signals of $35 \mathrm{kV}$ XLPE cable under different ground distances.

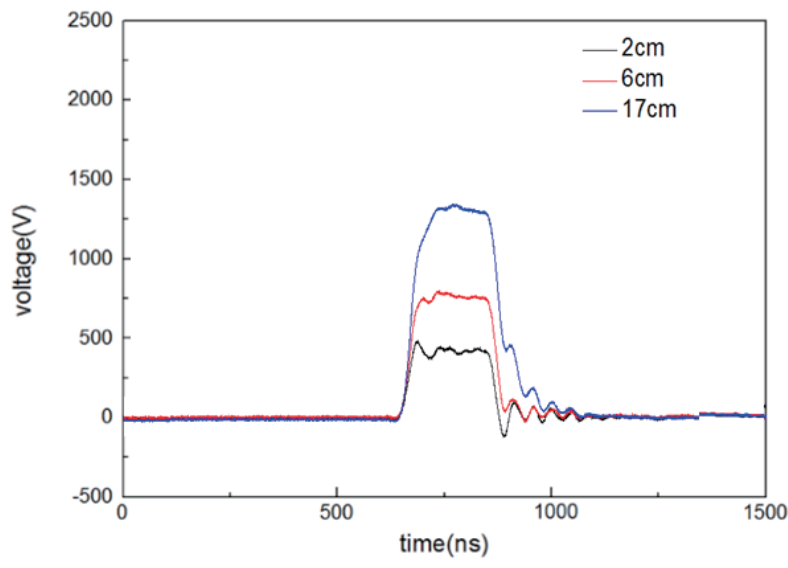

(a)

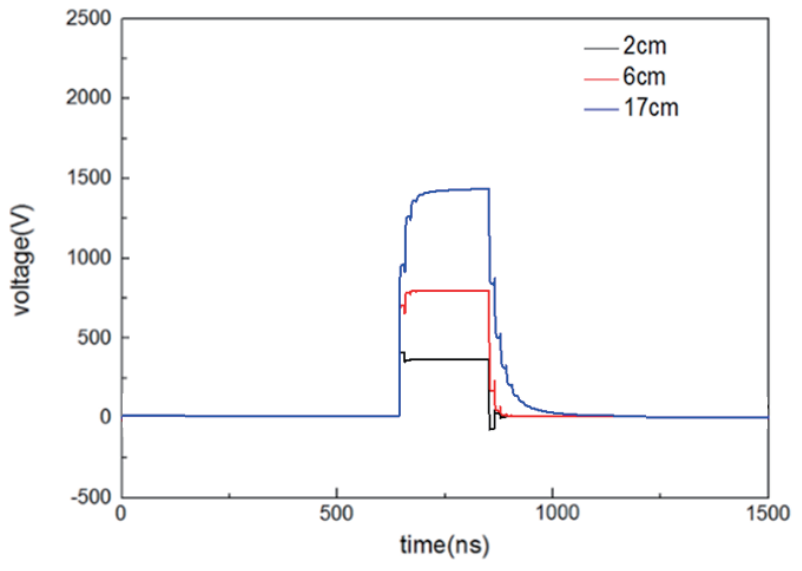

(b)

Fig. 8. (Color online) Coupled pulses of $35 \mathrm{kV}$ XLPE cable under different ground distances. (a) Test results. (b) Simulation results.

\subsection{Relationship between amplitude of coupled pulse and cable specification}

As mentioned above, the amplitude of the coupled pulse changes with the variation of the ground distance between the ground point and the measuring point. Moreover, the equivalent impedance of the coaxial cable also changes with the different cable specifications.

Figure 9(a) shows the coupled pulse waveforms of the 10, 35, and $110 \mathrm{kV}$ cables. Here, the ground distance is fixed at $17 \mathrm{~cm}$. It can be seen from Fig. 9(a) that the amplitude of the coupled pulse decreases with increasing voltage class. For the 10 and $35 \mathrm{kV}$ cables, the amplitudes of the coupled pulse are about 1.9 and $1.25 \mathrm{kV}$, while that of the coupled pulse for the $110 \mathrm{kV}$ cable suddenly dropped to $200 \mathrm{~V}$. Here, the voltage of the pulse source in the experiment and simulation is $2 \mathrm{kV}$. Figure 9(b) shows the simulation results of the coupled pulse according to Fig. 4 and the cable parameters in Table 1. It can be found that the test results in Fig. 9(a) are basically similar to the simulation results in Fig. 9(b). 


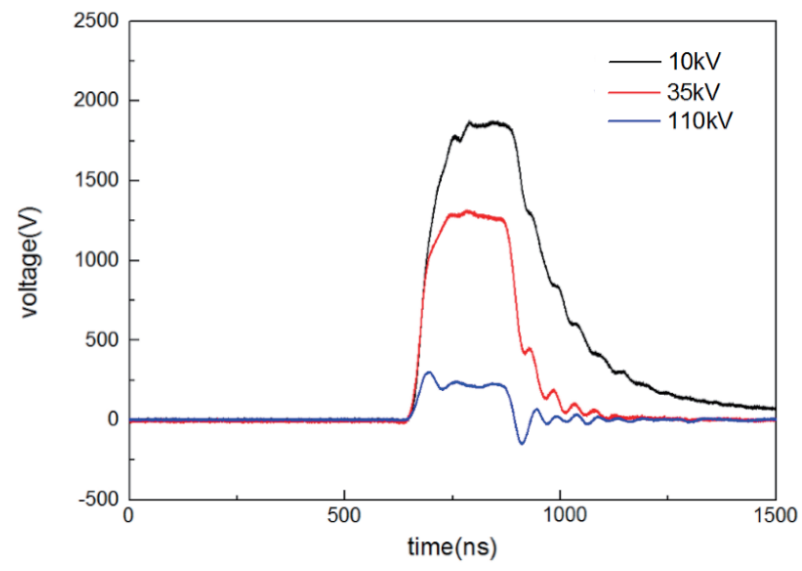

(a)

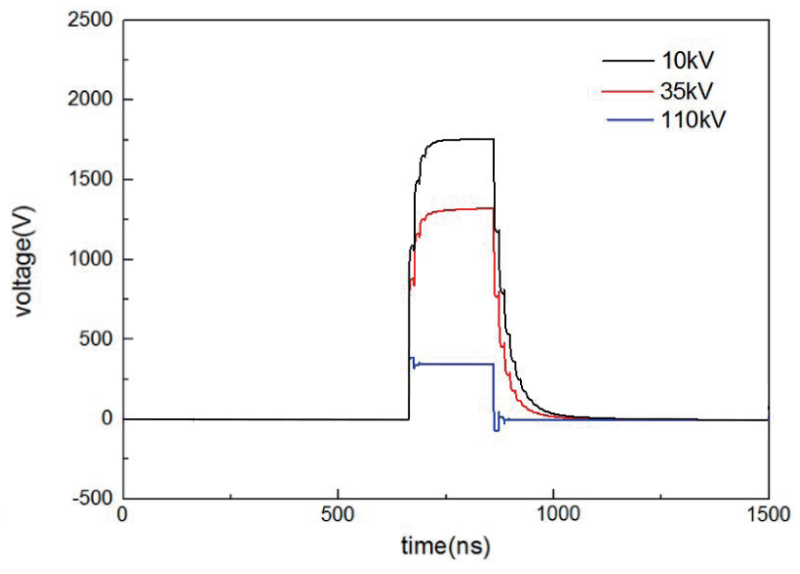

(b)

Fig. 9. (Color online) Coupled pulses of different cable specifications at a fixed ground distance of $17 \mathrm{~cm}$. (a) Test results. (b) Simulation results.

From the foregoing, the amplitude of the coupled pulse is also closely related to the cable's voltage class. At the same ground distance, the higher the cable's voltage class, the lower the resistance of the outer SC layer [see Table 1 or Eq. (2)], which directly results in a decrease in the amplitude of the coupled pulse.

\subsection{Relationship between coupled pulse and cable length}

Owing to the limit of the cable length measured in the laboratory, it is difficult to obtain the pulse waveform for the long cables. Hence, the amplitudes of the coupled pulse applied to the long cables were simulated by Pspice software.

Figure 10 shows the simulation results of the coupled pulse when the length of the $35 \mathrm{kV}$ cable changes from 1 to $50 \mathrm{~m}$. Here, the ground distance is also fixed at $17 \mathrm{~cm}$. As shown in Fig. 10, the amplitude of the coupled pulse is almost the same regardless of the cable length. In addition, a clear high-frequency oscillation appears on the pulse waveform, due to the impedance mismatch when the pulse spreads to the cable terminal and then reflects repeatedly. Moreover, the waveform oscillation weakens gradually with the increase in cable length.

\subsection{Relationship between coupled pulse and diameter of outer SC layer}

First, the electrical parameters of the 10, 35, and $110 \mathrm{kV}$ XLPE cables (referring to GB/T 12706 and GB/T 11017) were calculated using Eqs. (1) and (2), as shown in Table 2.

Figure 11 shows the simulation results of the relationship between the diameter of the outer SC layer and the pulse coupling efficiency. During the simulation, the cable length is $100 \mathrm{~m}$ and the ground distance is fixed at $17 \mathrm{~cm}$.

As shown in Fig. 11, the larger the diameter of the outer SC layer, the lower the pulse coupling efficiency. However, it is noteworthy that the pulse coupling efficiency is almost the same only if the cables have the same diameter of the outer SC layer (see Fig. 11 with the dotted ellipse). This means that the pulse coupling efficiency can be mainly determined by the diameter of the SC layer 


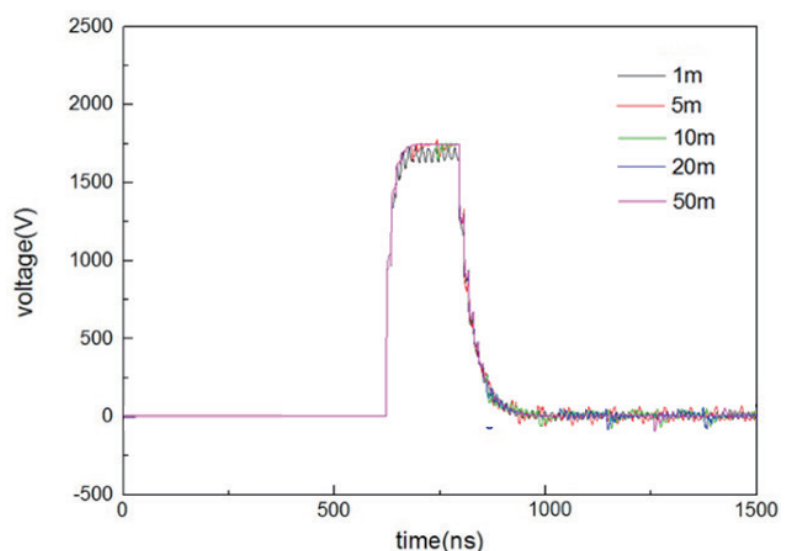

Fig. 10. (Color online) Coupled pulse waveforms of $35 \mathrm{kV}$ XLPE cable at different cable lengths (ground distance is fixed at $17 \mathrm{~cm}$ ).

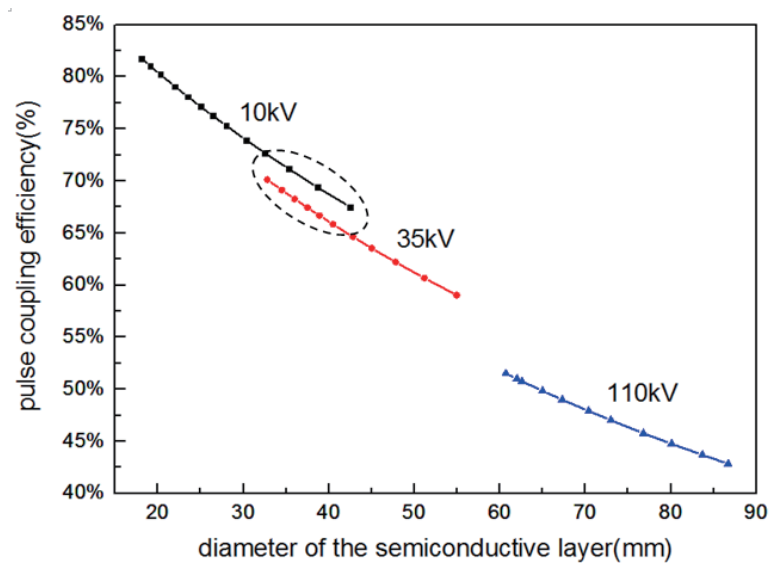

Fig. 11. (Color online) Pulse coupling efficiency under different cable specifications at a fixed ground distance of $17 \mathrm{~mm}$ (cable length is $100 \mathrm{~cm}$ ).

Table 2

Electrical parameters of actual cables at $10 \mathrm{MHz}$.

\begin{tabular}{lccc}
\hline \multirow{2}{*}{ Item } & \multicolumn{3}{c}{ Cable } \\
\cline { 2 - 4 } & $10 \mathrm{kV}$ & $35 \mathrm{kV}$ & $110 \mathrm{kV}$ \\
\hline Section $\left(\mathrm{mm}^{2}\right)$ & $25-630$ & $50-630$ & $240-1200$ \\
Diameter of outer SC layer $(\mathrm{mm})$ & $18.1-42.46$ & $32.8-54.96$ & $60.7-86.7$ \\
Capacitance of cable insulation $(\mathrm{pF} / \mathrm{m})$ & $157.1-498.0$ & $110.4-249.5$ & $123.3-269.5$ \\
Impedance of outer SC layer $(\mathrm{k} \Omega / \mathrm{m})$ & $5.4-13.1$ & $3.7-6.2$ & $1.9-2.7$ \\
\hline
\end{tabular}

regardless of the voltage class. In addition, the relationship between the diameter of the outer SC layer and the ground distance under different pulse coupling efficiencies is plotted in Fig. 12. In other words, the ground distance should be adjusted according to the diameter of the cable's outer SC layer, so as to ensure a constant pulse coupling efficiency.

\section{Analysis and Discussion}

\subsection{Equivalent circuit of pulse injection}

Simplifying Fig. 4(b), the lumped circuit of pulse injection from the outer SC layer can be redrawn, as shown in Fig. 13. Here, the pulse source is replaced by an ideal pulse source and an internal resistance $R$. $C_{x}$ is the capacitance of cable insulation at the measuring point, $C_{0}{ }^{\prime}$ is the lumped capacitance of cable insulation except the capacitance $C_{x}$ at the measuring point, and $R_{g}{ }^{\prime}$ is the resistance of the outer SC layer along the ground distance.

As shown in Fig. 13, when the pulse injects from the outer SC layer, the resistance of the outer SC layer and the capacitance of the cable insulation are connected in parallel and then connected in series with the internal resistance of the pulse source, so the amplitude of the coupled pulse can be approximately calculated according to Fig. 13, as shown in Eq. (3): 


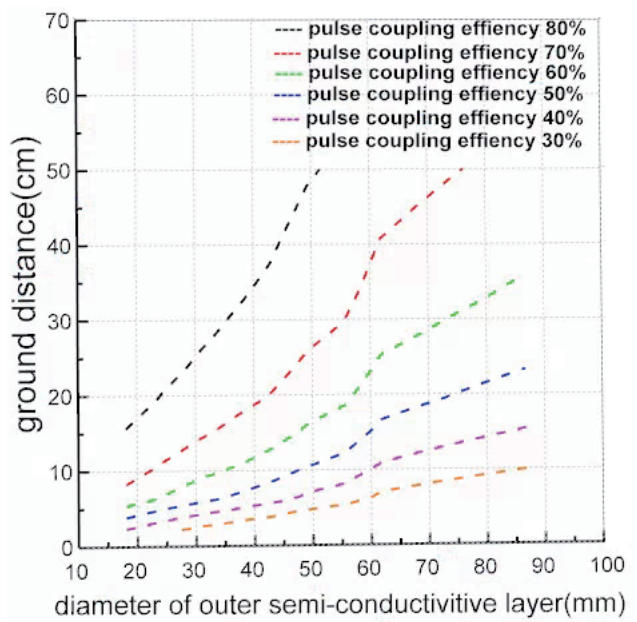

Fig. 12. (Color online) Relationship between the diameter of the outer SC layer and the ground distance under different pulse coupling efficiencies.

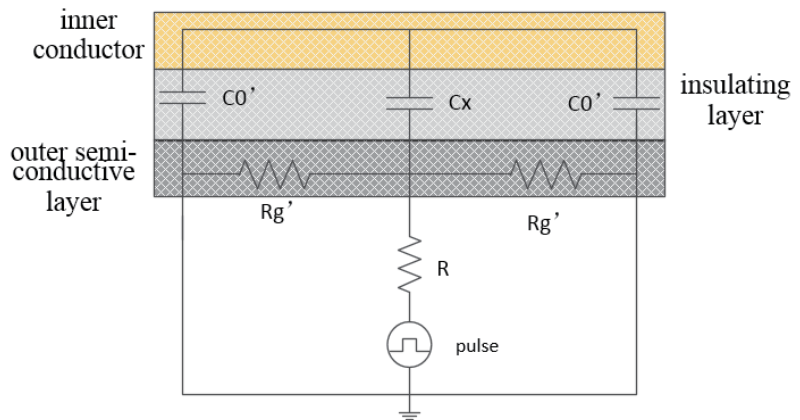

Fig. 13. (Color online) Lumped circuit diagram of pulse injection from the outer SC layer.

$$
\begin{array}{r}
U_{C_{x}}=U_{\text {pulse }} \times \frac{\frac{1}{2} R_{g}{ }^{\prime} / /\left(\frac{1}{j \omega C_{x}}+\frac{1}{j \omega 2 C_{0}{ }^{\prime}}\right)}{R+\frac{1}{2} R_{g}{ }^{\prime} / /\left(\frac{1}{j \omega C_{x}}+\frac{1}{j \omega 2 C_{0}{ }^{\prime}}\right)} \times \frac{\frac{1}{j \omega C_{x}}}{\frac{1}{j \omega 2 C_{0}{ }^{\prime}}+\frac{1}{j \omega C_{x}}} \\
=U_{\text {pulse }} \times \frac{1}{\frac{1}{\frac{1}{2} R_{g^{\prime}} / /\left(\frac{1}{j \omega C_{x}}+\frac{1}{j \omega 2 C_{0}}\right)}+1} \times \frac{1}{\frac{C_{x}}{C_{0}{ }^{\prime}}+1}
\end{array}
$$

For long cables, the capacitance $C_{x}$ at the measuring point is much lower than the cable's bulk capacitance $C_{0}{ }^{\prime}$. That is, $C_{0}{ }^{\prime} \gg C_{x}$. In addition, the capacitance $C_{x}$ is in picofarad and the maximal frequency of the nanosecond pulse with a width of $200 \mathrm{~ns}$ is in megahertz, so the impedance of $1 / j \omega C_{x}$ is much higher than the resistance of the outer SC layer $R_{g}{ }^{\prime}$. Thus, Eq. (3) can be simplified as

$$
U_{C_{x}} \approx U_{\text {pulse }} \times \frac{R_{g}{ }^{\prime}}{2 R+R_{g}{ }^{\prime}} .
$$

Therefore, the series circuits of the resistance of the outer SC layer $R_{g}{ }^{\prime}$ and the internal resistance of the pulse source $R$ mainly determine the amplitude of the injected pulse. In addition, as we have known, the thickness of the cable's outer SC layer almost ranges from 0.7 to $1.9 \mathrm{~mm}$ regardless of the cable specification. Thus, the additive term of $r_{3}+r_{2}$ in Eq. (2) determined by the diameter of the cable's outer SC layer will largely affect the equivalent resistance of the outer $\mathrm{SC}$ layer. This means that the longer the ground distance or the smaller the diameter of the cable's outer SC layer, the higher the resistance of the outer SC layer. As a result, the higher resistance of the outer SC layer, $R_{g}{ }^{\prime}$, in Eq. (4) will contribute to the higher amplitude of the coupled pulse (see Figs. 9, 10, and 13). 


\subsection{Propagation and reflection of pulse in cable}

In Fig. 10, a clear high-frequency oscillation appears on the coupled pulse, which is due to the impedance mismatch when the pulse spreads to the cable terminal and then reflects repeatedly. ${ }^{(20,21)}$ If the pulse injection point is located in the middle of the cable, the propagation of the pulse along the cable conductor can be illustrated in Fig. 14.

As shown in Fig. 14, the pulse propagates along both directions of the cable at the same time, and then is reflected back and forth at the cable terminal due to the impedance mismatch. Moreover, owing to the middle position at the measuring point, the reflection waves at both directions will be superimposed at the midpoint. The oscillation frequency in Fig. 10 is equal to the ratio of the electromagnetic wave velocity propagating in the cable to the cable length.

When the pulse propagates along the cable, the pulse voltage $U(x)$ along the cable is satisfied:

$$
U(x)=U_{C_{0}} e^{-\gamma x}
$$

Here, $U_{C_{0}}$ is the pulse voltage coupled with the cable capacitance $C_{0}$ and $\gamma$ is the coefficient of the attenuation and dispersion, which is determined by the cable's bulk capacitance and resistance.

By superimposing the multiple reflection waves at the pulse injection point, the coupled pulse accompanying with oscillation is formed as

$$
U_{p}=U_{C_{x}}+2 \sum_{k=1}^{\infty} U_{C_{0}} e^{-\gamma k L} .
$$

Here, $U_{C_{x}}$ is the pulse voltage coupled with the capacitance $C_{x}$ at the pulse injection point and $L$ is the cable length.

As mentioned in Sect. 4.1, we know that $C_{0} \gg C_{x}$. This means that $U_{C_{0}} \ll U_{C_{x}}$. Thus, the superimposed pulse voltage $U_{p}$ is approximately equal to the coupled pulse voltage $U_{C_{x}}$ in Eq. (6). Therefore, the high-frequency oscillation superposing on the coupled pulse hardly changes the amplitude of the coupled pulse. Furthermore, the longer cable facilitates to decrease the oscillation frequency and the higher bulk capacitance contributes to a smoother pulse wave (see Fig. 10).

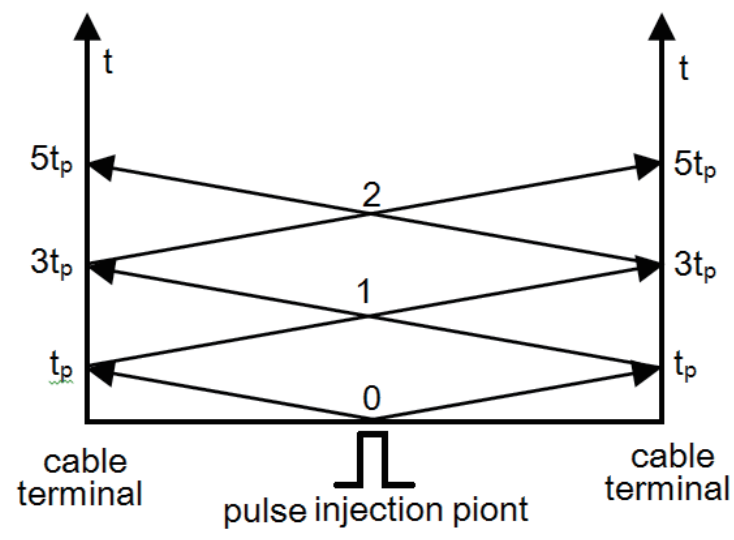

Fig. 14. Pulse propagation process along the cable. 


\section{Conclusions}

In this study, the original charge acoustic signals in a $35 \mathrm{kV}$ XLPE cable under different ground distances were measured using the PEA measurement system with pulse injection from a measuring electrode. At the same time, the effects of ground distance, cable specification and cable length on the coupled pulse waveform were studied through experiments and simulations. The following conclusions may be drawn from the analysis results:

(1) The amplitude of the coupled pulse at the measuring point is directly proportional to the ground distance along the outer SC layer but inversely proportional to the cable cross section or voltage class.

(2) The pulse coupling efficiency is almost the same only if the cables have the same diameter of the outer SC layer, regardless of the cable's voltage class.

(3) The longer cables, without changing the amplitude of the coupled pulse, facilitate to weaken the high-frequency oscillation superimposed on the pulse waveform.

(4) A constant pulse coupling efficiency can be obtained by adjusting the ground distance according to the diameter of the cable's outer SC layer.

\section{Acknowledgments}

The authors wish to thank the National Science Foundation of China under Project No. 51377131 and the State's Key Project of Research and Development Plan (Grant no. 2016YFB0900700).

\section{References}

1 L. Stendius and K. Eriksson: PowerGen Conference (Singapore, 1999).

2 V. G. Agelidis and L. Xu: Cigre Int. Conf. Power Systems (2001) p. 380.

3 L. Weimers: IEEE Power Eng. Rev. (1998) p. 19.

4 G. Damamme and C. L. Gressus: IEEE Trans. Dielectr. Electr. Insul. 4 (1997) 558.

5 K. S. Suh, J. H. Koo, and S. H. Lee: IEEE Trans. Dielectr. Electr. Insul. 3 (1996) 153.

6 A. Cavallini, G. C. Montanari, and F. Palmieri: IEEE Int. Conf. Dielectric Liquids (2005) p. 409.

7 K. C. Kao: IEEE Dielectrics and Electrical Insulation Society, Proc. 6th Int. Conf. Properties and Applications of Dielectric Materials Vol. 1 (2000) p. 1.

8 Y. W. Zhang, J. Lewiner, and C. Alquie: IEEE Trans. Dielectr. Electr. Insul. 3 (1996) 778.

9 T. Tanaka and A. Greenword: IEEE Trans. Power Syst. 95 (1978) 1749.

10 G. Blaise and C. L. Gressus: High Voltage Vacuum Insulation: Basic Concepts and Technological Practice (Rod Latham, London, 1995) p. 329.

11 S. Boggs: IEEE Electr. Insul. Mag. 20 (2004) 22.

12 K. Fukunaga, H. Miyata, and T. Takahashi: Proc. 3rd Int. Conf. Polymer Insulated Power Cables (1991) p. 520 .

13 M. Fu, G. Chen, and A. E. Davies: Proc. 6th Int. Conf. Properties and Applications of Dielectric Materials (2000) p. 104.

14 T. Takada: IEEE Power Engineering Society Winter Meeting (2000) p. 1609.

15 R. Liu and T. Takada: J. Phys D: Appl. Phys. 26 (1993) 986.

16 B. Vissouvanadin, T. T. N. Vu, and L. Berquez: IEEE Trans. Dielectr. Electr. Insul. 21 (2014) 821.

17 N. Hozumi, T. Okamoto, and T. Imajo: IEEE Int. Symp. Electrical Insulation (1992) p. 294.

18 X. Wang, J. Z. Xiong, and J. Q. Hao: Int. Conf. Condition Monitoring and Diagnosis (2016) p. 753.

19 R. Heinrich, S. Bonisch, D. Pommerenke, R. Jobava, and W. Kalkner: Eighth Int. Conf. (IEE Conf. Publ. No. $4732000)$ p. 212.

20 G. Mazzanti, G. Chen, and J. C. Fothergill: IEEE Trans. Dielectr. Electr. Insul. 22 (2015) 21.

21 T. Takada: IEEE Trans. Dielectr. Electr. Insul. 6 (1999) 519. 